hep-th/0604169

ITFA-2006-18

\title{
Holographic Coulomb branch vevs
}

\author{
Kostas Skenderis and Marika Taylor \\ Institute for Theoretical Physics, University of Amsterdam, \\ Valckenierstraat 65, 1018XE Amsterdam, The Netherlands \\ skenderi, taylor@science.uva.nl
}

\begin{abstract}
$\underline{\text { ABSTRACT }}$
We compute holographically the vevs of all chiral primary operators for supergravity solutions corresponding to the Coulomb branch of $\mathcal{N}=4 \mathrm{SYM}$ and find exact agreement with the corresponding field theory computation. Using the dictionary between 10d geometries and field theory developed to extract these vevs, we propose a gravity dual of a half supersymmetric deformation of $\mathcal{N}=4 \mathrm{SYM}$ (on $M i n k_{4}$ ) by certain irrelevant operators.
\end{abstract}




\section{Introduction}

Since its formulation [1]-[3] there have been numerous tests of the AdS/CFT duality, see $[4,5]$ for reviews. Soon afterward the duality was extended to non-conformal field theories obtained by deformations or vevs of the original CFT. In such cases the duality involves an asymptotically AdS spacetime. However, quantitative tests of the correspondence in this more general set up are rather scarce.

Perhaps one of the simplest cases to explore is that of the Coulomb branch of the $\mathcal{N}=4$ SYM theory. The theory still possesses 16 supercharges and supersymmetry protects the vevs from quantum corrections. This is thus an ideal case for testing gravity/gauge theory duality away from the conformal point. Although it has long been recognized that there is a one to one correspondence between (the near-horizon limit of) multicenter D3 brane solutions and the $\mathrm{CB}$ of $\mathcal{N}=4 \mathrm{SYM}[6]$ a precise gravitational computation of the vevs was never done (apart from in the specific cases reviewed below). These solutions are determined by a harmonic function and Klebanov and Witten proposed in [7] that the vevs can be extracted from the harmonic function. The results of this paper confirm that expectation.

More quantitative progress has been achieved for the specific case of a distribution of D3 branes on a disc. In this case there is an associated solution of the five dimensional gauged supergravity [8] and using the technology of holographic renormalization [9] the expectation values (and 2-point functions) of the stress energy tensor and of a dimension two gauge invariant operator were computed and shown to agree with field theory expectations in $[10,11]$.

The restriction to this subsector of operators was due to starting from the five dimensional supergravity solution. More recently in [12] we have developed a precise holographic map that allows one also to treat operators dual to fields that are not part of the five dimensional gauged supergravity. Applying this map to the corresponding ten dimensional supergravity solution we additionally computed the vev of a dimension 4 operator and again found exact agreement with field theory. This was the first quantitative computation of a vev of an operator dual to field that is not part of the $5 d$ gauged supergravity.

In this letter we essentially solve the problem in its most general form. We consider a point in the $\mathrm{CB}$ of $\mathcal{N}=4 \mathrm{SYM}$ characterized in the large $N$ limit by a general distribution of eigenvalues and we show that the gravitationally computed vevs of all gauge invariant operators are in exact agreement with the field theory answer. We should note, however, there is still an open technical issue regarding the cancellation of certain terms that was 
checked only for a specific case (see the discussion in section 5).

Finally we point out that one can include non-normalizable terms in the harmonic function appearing in the supergravity solution for separated D3-branes and these can also be interpreted in AdS/CFT. Such terms correspond to deformations of the SYM theory by half supersymmetric irrelevant operators of the form $\operatorname{Tr}\left(F^{4} X^{k}\right)$.

The paper is organized as follows. In the next section we discuss the field theory side of the story. In section 3 we review the Coulomb branch solutions; in section 4 we summarize the results from [12] whilst section 5 contains the holographic computation of the vevs for the general case. In section 6 we propose a gravity dual for certain deformations of $\mathcal{N}=4$ SYM preserving 16 supercharges. Appendix A contains a proof of the addition theorem that is used in the extraction of vevs in section 5 .

\section{$2 \mathcal{N}=4$ SYM on the Coulomb branch}

$\mathcal{N}=4$ SYM contains 6 scalar fields $X^{i_{1}}$ in the adjoint representation of the gauge group that we take to be $S U(N)$. The Coulomb branch (CB) of $\mathcal{N}=4$ SYM corresponds to giving a vacuum expectation value (vev) to the scalars subject to the condition $\left[X^{i_{1}}, X^{i_{2}}\right]=0$. A useful parametrization of the $\mathrm{CB}$ branch is in terms of vevs of composite operators. The relevant operators here are the chiral primaries (CPOs),

$$
\mathcal{O}^{I_{1}}=\mathcal{N}_{I_{1}} C_{i_{1} \cdots i_{k}}^{I_{1}} \operatorname{Tr}\left(X^{i_{1}} \cdots X^{i_{k}}\right)
$$

where $C^{I_{1}}$ is a totally symmetric traceless rank $k$ tensor of $S O(6)$ which is normalized such that $\left\langle C^{I_{1}} C^{I_{2}}\right\rangle=C_{i_{1} \cdots i_{k}}^{I_{1}} C_{i_{1} \cdots i_{k}}^{I_{2}}=\delta^{I_{1} I_{2}}$ and $\mathcal{N}_{I_{1}}$ is a normalization factor. We choose this factor such that the normalization of the 2-point functions computed in field theory and in supergravity is the same

$$
\mathcal{N}_{I_{1}}=\frac{N}{\pi^{2}} 2^{k / 2}(k-2) \sqrt{\frac{k-1}{k}}\left(\frac{2 \pi^{2}}{\lambda}\right)^{k / 2} ; \quad k \neq 2,
$$

where $\lambda$ is the 't Hooft coupling. Note the field theory computation of the 2-point functions is done in the large $N$ limit and with canonically normalized scalars. The $k=2$ case is obtained by replacing the factor of $(k-2)$ by 2 .

We now consider an arbitrary smooth unit normalized distribution of eigenvalues, $\rho(x)$, where $x$ parametrizes $R^{6}$. In the large $N$ limit the trace may be evaluated via such a continuous eigenvalue distribution

$$
\left\langle C_{i_{1} \cdots i_{k}}^{I} \operatorname{Tr}\left(\mathrm{X}^{\mathrm{i}_{1}} \cdots \mathrm{X}^{\mathrm{i}_{\mathrm{k}}}\right)\right\rangle=N \int d^{6} x \rho(x)\left(C_{i_{1} \cdots i_{k}}^{I} x^{i_{1}} \cdots x^{i_{k}}\right)
$$


giving the following formula for the vevs

$$
\left\langle\mathcal{O}^{I_{1}}\right\rangle=\frac{N^{2}}{\pi^{2}} 2^{k / 2}(k-2) \sqrt{\frac{k-1}{k}}\left(\frac{2 \pi^{2}}{\lambda}\right)^{k / 2} \int d^{6} x \rho(x)\left(C_{i_{1} \cdots i_{k}}^{I} x^{i_{1}} \cdots x^{i_{k}}\right), \quad k \neq 2,
$$

where again for $k=2$ one replaces the factor of $(k-2)$ by 2 . The aim of this paper is to reproduce this formula from supergravity for all $k$ and general eigenvalue distributions.

Before proceeding let us briefly review the properties of a uniform distribution of eigenvalues of $X^{1}$ and $X^{2}$ on a disc of radius $a$ and vanishing vev for the remaining scalars, $\left\langle X^{3}\right\rangle=\left\langle X^{4}\right\rangle=\left\langle X^{5}\right\rangle=\left\langle X^{6}\right\rangle=0$. In this case the vevs break the $\mathrm{R}$ symmetry from $S O(6)$ to $S O(2) \times S O(4)$. This example was recently discussed in some detail in [12] and the corresponding vevs were shown to be equal to

$$
\left\langle\mathcal{O}^{2 n}\right\rangle=\frac{\mathcal{N}_{2 n} a^{2 n}}{2^{n} \sqrt{2 n+1}} N
$$

where the operators here are the singlets under the decomposition of $S O(6)$ into $S O(2) \times$ $S O(4)$.

\section{Coulomb branch solutions}

A generic Coulomb branch solution describing a distribution of D3-branes is given by:

$$
\begin{aligned}
d s^{2} & =H\left(x_{\perp}\right)^{-1 / 2} d x_{\|}^{2}+H\left(x_{\perp}\right)^{1 / 2} d x_{\perp}^{2} \\
F & =\frac{1}{4}\left(d H^{-1} \wedge \omega_{\|}-*_{\perp} d_{\perp} H\right)
\end{aligned}
$$

where $\omega_{\|}$is the volume form in the (flat) worldvolume directions and $*_{\perp}$ and $d_{\perp}$ refer to the Hodge star and exterior derivative in the flat transverse directions and $H$ is a harmonic function,

$$
\square H=0 .
$$

For the solution to be asymptotically AdS, $H$ should behave to leading order as $r^{-4}$ when $r \rightarrow \infty$, where $r$ is the radial coordinate of the flat overall transverse direction. The most general solution of (3.2) with these boundary conditions is

$$
H=\sum_{k, I} h_{k I} \frac{Y_{k}^{I}(\vec{\theta})}{r^{k+4}}
$$

where the coordinates on the flat $R^{6}$ are $(r, \vec{\theta})$ with $\vec{\theta}$ labeling the coordinates on the $S^{5}$. $Y_{k}^{I}$ is a normalized spherical harmonic of degree $k$ with $I$ labeling its remaining quantum numbers; normalized as

$$
\int_{S^{5}} Y_{k_{1}}^{I_{1}} Y_{k_{2}}^{I_{2}}=\pi^{3} \frac{\delta^{I_{1} I_{2}} \delta_{k_{1} k_{2}}}{2^{k_{1}-1}\left(k_{1}+1\right)\left(k_{1}+2\right)} \equiv \delta^{I_{1} I_{2}} z_{k_{1}} .
$$


The leading order term is given by $h_{00}=L^{4}=4 \pi g_{s} N\left(\alpha^{\prime}\right)^{2}$ if the total number of D3-branes is to be $N$, whilst by measuring distances from the centre of mass one can as usual choose the $k=1$ terms to vanish. We will discuss more general boundary conditions for $H$ in section 6 .

The harmonic function corresponding to a source distribution of D3-branes $\rho(x)$ (normalized to one) is given by

$$
H=L^{4} \int d^{6} y \frac{\rho(y)}{|x-y|^{4}},
$$

We show in appendix A that the asymptotics of this harmonic function can be written as in (3.3) with coefficients

$$
h_{k I}=2^{k}(k+1) L^{4} \int d^{6} x \rho(x)\left(C_{i_{1} \cdots i_{k}}^{I} x^{i_{1}} \cdots x^{i_{k}}\right)
$$

where $C_{i_{1} \cdots i_{k}}^{I}$ is totally symmetric and traceless and the basis of $C^{I}$ is orthonormal. Comparing (3.6) with (2.4) we see that these coefficients are proportional to the vevs of the CPOs.

In the case of a uniform distribution of D3 branes on a disc of radius $l$ one can straightforwardly do the integral in (3.6) [12] with the result being

$$
h_{2 n 0}=L^{4} 2^{n} \sqrt{2 n+1} l^{2 n}
$$

where $I=0$ signifies that only harmonics that are singlets under the decomposition $S O(6) \rightarrow S O(2) \times S O(4)$ are involved.

Finally let us comment on the case of a spherical shell of D3 branes of radius $R$, for which the harmonic function is [6]

$$
H=\frac{L^{4}}{r^{4}}, \quad r \geq R ; \quad H=\frac{L^{4}}{R^{4}}, \quad r<R,
$$

so the geometry is flat within the shell. The asymptotics of this harmonic function are trivially of the form (3.3), with the absence of any perturbation relative to AdS corresponding to the fact that there are no vevs for CPOs, since there are no $S O(6)$ singlet CPOs. The interpretation of constant terms in the harmonic function will be discussed in section 6 .

\section{General method for extracting vevs}

In this section we will give a brief review of the methods developed in [12] for extracting vevs from a given asymptotically $A d S_{5} \times S^{5}$ geometry. The first step is to write the solution 
as the $A d S_{5} \times S^{5}$ solution plus a deviation ${ }^{1}$

$$
\begin{aligned}
g_{M N} & =g_{M N}^{o}+h_{M N} \\
F_{M N P Q R} & =F_{M N P Q R}^{o}+f_{M N P Q R}
\end{aligned}
$$

where $g_{M N}^{o}$ and $F_{M N P Q R}^{o}$ are the metric and 5-form of the $A d S_{5} \times S^{5}$ solution. (The solutions under consideration here do not involve the other supergravity fields, so we restrict our discussion to the metric and five form.) Next we expand the perturbations $h_{M N}, f_{M N P Q R}$ in $S^{5}$ harmonics. The general expansion is given in [12]; here we only quote the following two components since these will be useful later:

$$
\begin{aligned}
h_{a}^{a}(x, y) & =\sum \pi^{I_{1}}(x) Y^{I_{1}}(y) \\
f_{a b c d e}(x, y) & =\sum b^{I_{1}}(x) \Lambda^{I_{1}} \epsilon_{a b c d e} Y^{I_{1}}(y)
\end{aligned}
$$

where $Y^{I_{1}}$ are scalar spherical harmonics and $\Lambda^{I_{1}}$ is the eigenvalue of the scalar harmonic under (minus) the d'Alembertian. It is convenient to introduce the following linear combinations of $\pi^{I_{1}}$ and $b^{I_{1}}$

$$
s^{I_{1}}=\frac{1}{20(k+2)}\left(\pi^{I_{1}}-10(k+4) b^{I_{1}}\right), \quad t^{I_{1}}=\frac{1}{20(k+2)}\left(\pi^{I_{1}}+10 k b^{I_{1}}\right),
$$

since these combinations are mass eigenstates of the linearized field equations around $A d S_{5} \times$ $S^{5}[13]$. The $s^{I_{1}}$ fields correspond to the chiral primary operators in (2.1) and the $t^{I_{1}}$ fields to half supersymmetric operators of the schematic form $\operatorname{Tr} F^{4} X^{k}$.

There are three ingredients that enter into the map from coefficients of the harmonic expansion in (4.2) to vevs in the dual QFT. The first is the construction of gauge invariant variables, if the solution is not in de Donder gauge (which indeed is often not a convenient gauge choice for the asymptotic expansion). De Donder gauge, $D^{a} h_{(a b)}=D^{a} h_{a \mu}=0$, means that the harmonic expansion of the metric deviations does not involve terms with derivatives of the harmonics. It is easy to see that the $\mathrm{CB}$ metrics (3.1) in the coordinate system where (3.3) holds satisfy this requirement (but notice that the CB solutions expressed in the coordinates of [8] are not in de Donder gauge, as discussed in [12]). So since we can conveniently work in de Donder gauge here, we do not need to review the construction of gauge invariant variables (and we simplify our notation relative to [12] by dropping the tildes and hats from our notation).

The second ingredient is the non-linear KK map from ten dimensional fields to five dimensional ones. For simplicity let us restrict to the subsector involving $s^{2}$ and $s^{4}$ fields

\footnotetext{
${ }^{1}$ We follow the conventions of [12]. Our index conventions are: $M, N, \ldots$ are $10 d$ indices, $\mu, \nu, \ldots$ are $A d S_{5}$ indices, $a, b, \ldots$ are $S^{5}$ indices. $x$ denotes AdS coordinates and $y S^{5}$ coordinates.
} 
that are singlets under $S O(6) \rightarrow S O(2) \times S O(4)$. The non-linear reduction map to second order in the fields reads [14]

$$
\begin{aligned}
& S^{2}=\frac{\sqrt{8}}{3}\left(s^{2}-\frac{2 \sqrt{3}}{15}\left(s^{2}\right)^{2}-\frac{1}{12 \sqrt{12}} D_{\mu} s^{2} D^{\mu} s^{2}\right) \\
& S^{4}=\frac{2 \sqrt{3}}{5}\left(s^{4}-\frac{83}{18 \sqrt{5}}\left(s^{2}\right)^{2}-\frac{7}{18 \sqrt{5}} D_{\mu} s^{2} D^{\mu} s^{2}\right)
\end{aligned}
$$

where capital letters denote five dimensional fields and small letters ten dimensional fields. The fields $S^{2}$ and $S^{4}$ defined by (4.5) solve the five dimensional equations up to second order

$$
\square S^{2}=-\frac{4}{\sqrt{6}}\left(S^{2}\right)^{2}, \quad \square S^{4}=0 .
$$

In (4.5)-(4.6) we have included only terms that can potentially contribute to the asymptotic expansion of $S^{2}$ and $S^{4}$ up to the order required for extraction of vevs. More generally to extract the vev of the operator dual to $S^{\Delta}$ one would need to retain terms involving up to $\Delta / 2$ fields. The overall field normalization is chosen such that in the $5 d$ action, all fields are canonically normalized, apart from an overall factor of $N^{2} / 2 \pi^{2}$.

The final step is to use the method of holographic renormalization to extract the vevs from the asymptotics of the $5 d$ fields. This is by now a standard procedure (see [15] for a review), except that here one needs to include additional terms to accommodate extremal couplings (see section 5.4 of [12]). The relation between field asymptotics and vevs is most transparent in Hamiltonian variables where the radius plays the role of time. The 1-point functions are then related to the radial canonical momenta of the bulk fields [16]. For the operators $\mathcal{O}^{2}$ and $\mathcal{O}^{4}$ (which are singlets under $S O(6) \rightarrow S O(2) \times S O(4)$ ) the relations are $[12]:$

$$
\begin{aligned}
\left\langle\mathcal{O}^{4}\right\rangle & =\pi_{(2)}^{2} \\
\left\langle\mathcal{O}^{4}\right\rangle & =\pi_{(4)}^{4}+\frac{3 \mathcal{N}_{4}}{\sqrt{5} \mathcal{N}_{2}^{2} N}\left(\pi_{(2)}^{2}\right)^{2}
\end{aligned}
$$

where $\pi_{(k)}^{m}$ indicates the part of the canonical momentum of the field $S^{m}$ that scales with weight $k$ and $\mathcal{N}_{k}$ are the normalization factors given in (2.2). The relevant part of the canonical momenta can be expressed in terms of the asymptotic expansion of the $5 d$ fields as follows

$$
\pi_{(2 k-4)}^{k}=\frac{N^{2}}{2 \pi^{2}}(2 k-4)\left[S^{k}\right]_{k}
$$

where the notation $[A]_{k}$ indicates the coefficient of the $z^{k}$ term in $A$ and $z$ is the FeffermanGraham radial coordinate. The relation (4.8) holds for $k \neq 2$; when $k=2$ one should replaces the factor $(2 k-4)$ by 2 . 
For reasons which will become clear later it is useful to express the vevs directly in terms of the coefficients that appear in the $10 d$ solution. Using the results reviewed above one obtains

$$
\begin{aligned}
\left\langle\mathcal{O}^{2}\right\rangle & =\frac{N^{2}}{2 \pi^{2}} \frac{2 \sqrt{8}}{3}\left[s^{2}\right]_{2} \\
\left\langle\mathcal{O}^{4}\right\rangle & =\frac{N^{2}}{2 \pi^{2}} \frac{4 \sqrt{3}}{5}\left[2 s^{4}+\frac{37}{9 \sqrt{5}}\left(s^{2}\right)^{2}-\frac{7}{9 \sqrt{5}}\left(D s^{2}\right)^{2}\right]_{4}
\end{aligned}
$$

The expression for $\left\langle\mathcal{O}^{4}\right\rangle$ can be further simplified for solutions with $s^{2}$ only depending on the radial coordinate, such as those under consideration in this paper. In such cases, $\left[\left(D s^{2}\right)^{2}\right]_{4}=4\left[\left(s^{2}\right)^{2}\right]_{4}$ and we obtain

$$
\left\langle\mathcal{O}^{4}\right\rangle=\frac{N^{2}}{2 \pi^{2}} \frac{4 \sqrt{3}}{5}\left[2 s^{4}+\frac{1}{\sqrt{5}}\left(s^{2}\right)^{2}\right]_{4}
$$

The general method outlined here can be applied to extract the vevs of all other higher dimension operators. However, the procedure becomes complex as the operator dimension increases, since one has to retain terms to higher and higher order in the reduction map, the field equations and the relations which give the vevs. The purpose of the current paper is to point out that simplifications occur for the Coulomb branch solutions: for these we will be able to carry out the vev computation for arbitrarily high dimension operators.

\section{$5 \quad$ Obtaining the vevs}

We now return to the Coulomb branch solutions and express the harmonic function as

$$
H=\frac{L^{4}}{r^{4}}+\delta H,
$$

i.e. we separate the $k=0, I=0$ term in (3.3) that yields the $A d S_{5} \times S^{5}$ part of the solution from the remaining terms. The metric and five form field become

$$
\begin{aligned}
d s^{2} & =L^{2} r^{2}\left(1+\frac{r^{4} \delta H}{L^{4}}\right)^{-\frac{1}{2}} d x_{\|}^{2}+L^{2}\left(1+\frac{r^{4} \delta H}{L^{4}}\right)^{\frac{1}{2}}\left(\frac{d r^{2}}{r^{2}}+d \Omega_{5}^{2}\right) \\
F_{5} & =\left(L^{4}-\frac{1}{4} r^{5} \partial_{r} \delta H\right) d \Omega_{5}+\frac{1}{4} r^{3} d r \wedge *_{S^{5}} D_{a} \Delta H+\frac{1}{4} d\left(L^{4} r^{4}\left(1+\frac{\delta H r^{4}}{L^{4}}\right)^{-1}\right) \wedge \omega_{\|} .
\end{aligned}
$$

Note that the coordinates on $R^{3,1}$ have been rescaled as

$$
x_{\|} \rightarrow L^{2} x_{\|}
$$

so that the metric scales as $L^{2}$. 
This coordinate system is manifestly compatible with the de Donder gauge for fluctuations; we can immediately read off the following expression for the field $b_{k}^{I}$ in (4.2) as

$$
b_{k}^{I}=-\frac{h_{k I}}{4 L^{4} k r^{k}}
$$

whilst the trace of the fluctuation on the sphere is

$$
\pi=5\left(\left(1+\frac{r^{4} \delta H}{L^{4}}\right)^{1 / 2}-1\right) .
$$

Retaining only linear terms in the expansion of the square root gives

$$
\pi_{k}^{I}=\frac{5 h_{k I}}{2 L^{4} r^{k}}
$$

Note however that there is no justification generically for retaining only the linear term. Indeed the quadratic correction gives

$$
-\frac{5}{8 z(k)} \sum_{k_{1}, I_{1}, I_{2}} \frac{1}{L^{8} r^{k}} h_{k_{1} I_{1}} h_{\left(k-k_{1}\right) I_{2}}\left\langle Y_{k_{1}}^{I_{1}} Y_{\left(k-k_{1}\right)}^{I_{2}} Y_{k}^{I}\right\rangle,
$$

where the term in parenthesis indicates the triple overlap of the scalar harmonics. This term is not suppressed compared to the linear term (recall the $h_{k I}$ contain a factor of $L^{4}$ so the factors of $L$ cancel in (5.7)); there is no small parameter in general. The issue of non-liner terms first appears for dimension 4 operators. In this case we constructed the holographic map from first principles in [12], as reviewed in the previous section, so we should be able to understand whether the quadratic corrections contribute. Notice that since $h_{k I}$ is proportional to the vev of a chiral primary operator, the quadratic correction looks like a double trace contribution.

To understand this issue let us restrict to the special case of a disc distribution which was the case understood in detail in [12]. In this case $s^{2}$ and $s^{4}$ are given by

$$
s^{2}=\frac{1}{8} h_{20}, \quad s^{4}=\frac{1}{16} h_{40}-\frac{1}{128 \sqrt{5}}\left(h_{20}\right)^{2},
$$

where $h_{20}$ and $h_{40}$ are given in (3.7). The term in $s^{4}$ quadratic in $h_{20}$ comes from (5.7). Inserting these values in the expression for the vevs (4.11) we find that the $\left(h_{20}\right)^{2}$ part of $s^{4}$ is precisely cancelled by the $\left(s_{2}\right)^{2}$ term! The remaining terms are exactly right to reproduce (2.5). Notice that the $\left(s_{2}\right)^{2}$ terms in (4.11) originate from two sources. One is the non-linear terms in the KK map and the second are the non-linear terms in the 1-point function (4.7). Based on this result we conjecture that the same type of cancellation will occur for all operators in the general case. 
So now let us return to the general case and assume that such a cancellation takes place. We thus retain only the linear term (5.6) and ignore non-linear terms in the KK map and non-linear terms in the 1-point function. The 10d fluctuations under these assumptions are

$$
s_{k}^{I}=\frac{h_{k I}}{4 k L^{4} r^{k}}, \quad t_{k}^{I}=0,
$$

so the canonically normalized five dimensional fields are equal to

$$
S_{k}^{I}=\frac{\sqrt{(k-1)}}{2^{k / 2} \sqrt{k}(k+1)} \frac{h_{k I}}{L^{4} r^{k}}, \quad T_{k}^{I}=0,
$$

where for completeness we also quote the values of the $t$ field. This implies the following expression for the vev

$$
\left\langle\mathcal{O}_{k}^{I}\right\rangle=\frac{N^{2}}{2 \pi^{2}}(2 k-4)\left[S^{I}\right]_{k}=\frac{N^{2}}{\pi^{2}} \frac{(k-2) \sqrt{(k-1)}}{2^{k / 2} \sqrt{k}(k+1)} \frac{h_{k I}}{L^{4}},
$$

for $k \neq 2$ whilst the formula for $k=2$ follows using the replacement $(k-2) \rightarrow 2$. Using the identity (3.6) then gives a final expression for the vev as

$$
\left\langle\mathcal{O}_{k}^{I}\right\rangle=\frac{N^{2}}{\pi^{2}} 2^{k / 2}(k-2) \sqrt{\frac{k-1}{k}} \int d^{6} x^{\prime} \rho\left(x^{\prime}\right)\left(C_{i_{1} \cdots i_{k}}^{I}\left(x^{\prime}\right)^{i_{1}} \cdots\left(x^{\prime}\right)^{i_{k}}\right),
$$

for $k \neq 2$ with the analogous expression for $k=2$ being obtained by the replacement $(k-2) \rightarrow 2$. To match supergravity and field theory normalizations, field theory lengths must be scaled by a factor of $\sqrt{\lambda / 2 \pi^{2}}$. This is due to the rescaling in (5.3) and is explained in section 6.3 of [12]. Taking this factor into account we find exact agreement between the supergravity and field theory computations in (5.12) and (2.4)!

This result is highly non-trivial: whilst all the vevs must necessarily be encoded in the harmonic function, it is surprising that one can extract them so simply by the above linearization of the ten-dimensional fields. Note that this procedure for extracting the Coulomb branch vevs was suggested in [7], although the interpretation of the neglected terms was and remains unclear. Presumably the linearization can be rigorously justified in this case by proving that the non-linear terms cancel when one carries out the holographic renormalization procedure directly in ten dimensions. This issue is currently under investigation.

One would also not anticipate that there is an analogous route for extracting vevs from general asymptotically $A d S_{5} \times S^{5}$ solutions. In the CB example, the simplifications arose when we expanded the solution in a particular radial coordinate, which turned out to be exactly the Fefferman-Graham coordinate, and then linearized in a natural way. Had we expanded the same solutions in the coordinate systems of [8], we would have had no 
motivation for retaining the required "linearized" subset of terms in the perturbations. Indeed, in the discussion of the disk distribution in [12], only the complete gauge invariant fields $\pi_{k}^{I}$ played a role. For more general supergravity solutions, even those such as the bubbling geometries of [17] which are built from harmonic functions, it is not obvious how one would even define the above procedure or why it should be justified. By contrast, the method for extracting vevs presented in [12] applies to all asymptotically $A d S_{5} \times S^{5}$ solutions.

\section{Duals of $1 / 2$-susy deformations of $\mathcal{N}=4$ SYM}

In section 3 we discussed the supergravity solution (3.1) describing a distribution of D3branes. The harmonic function $H$ entering the solution was constrained to vanish as $r^{-4}$ in order for the solution to be asymptotically AdS. We discuss here solutions obtained by relaxing this condition, and their interpretation using the AdS/CFT dictionary, in particular using the map between ten dimensional fields and the gauge theory discussed in the previous section.

The most general solution of $(3.2)$ is

$$
H=\sum_{k, I}\left(l_{k I} r^{k}+\frac{h_{k I}}{r^{k+4}}\right) Y_{k}^{I}
$$

The case with only the $k=I=0$ terms is D3 brane solution with the asymptotically flat region included. The more general solution (6.1) still preserves 16 supercharges, and as we will shortly see, surprisingly admits an AdS/CFT interpretation despite the fact that the solution is not asymptotically AdS (in any usual usage of the word).

We proceed as in the previous section by writing

$$
H=\frac{L^{4}}{r^{4}}+\delta H
$$

and keeping only the linear term in $\delta H$. The terms proportional to $h_{k I}$ have been discussed already, so we only consider here the new terms proportional to $l_{k I}$. From the metric and five-form we read off

$$
\pi_{k}^{I}=\frac{5 l_{k I}}{2 L^{4}} r^{k+4}, \quad b_{k}^{I}=\frac{l_{k I}}{4(k+4) L^{4}} r^{k+4} .
$$

Forming the $s$ and $t$ combinations we obtain

$$
s_{k}^{I}=0, \quad t_{k}^{I}=\frac{l_{k I}}{4(k+4)} r^{k+4} .
$$


The radial behavior of the $t$ field is exactly right for the solution to correspond to a deformation of the $N=4$ SYM theory by the operators dual to the $t^{k}$ fields, with the deformation parameter being (proportional to) $l_{k I}$. The restriction of keeping only linear terms in $\delta H$ may be justified by considering the deformation parameters $l_{k I}$ to be small (although the results may hold more generally due to cancellations as in the case of $h_{k I}$ ).

Such a deformation was discussed in [18] where it was argued that the gauge beta function continues to vanish. This corresponds to the fact that the dilaton and axion are constant in the solutions we discuss. In the same paper, it was argued (extending earlier work $[19,20]$ ) that the full D3-brane solution (with the asymptotic flat region included) is dual to $N=4$ SYM deformed by a dimension 8 operator. This is precisely the operator dual to $t^{0}$. In this paper we generalize this proposal to the general case of a deformation by all operators dual to $t^{k}$. It is easy to check that all symmetries (susy, R-symmetry) match between the supergravity and field theory descriptions. Notice that the operators $t^{k}$ are irrelevant so one expects a strong backreaction on the asymptotics of the dual geometry. It would be interesting to explore this case further, especially since the geometry is far from being asymptotically AdS so one may learn about how holography works in more general contexts.

\section{Acknowledgments}

The authors are supported by NWO, KS via the Vernieuwingsimplus grant "Quantum gravity and particle physics" and MMT via the Vidi grant "Holography, duality and time dependence in string theory".

\section{A The addition theorem for $S O(6)$ spherical harmonics}

In this appendix we prove (3.6). The harmonic function

$$
H=L^{4} \int d^{6} y \frac{\rho(y)}{|x-y|^{4}},
$$

can be expanded in powers of $r=\sqrt{x^{2}}$ as

$$
\begin{aligned}
H & =\frac{L^{4}}{r^{4}} \int d^{6} y \rho(y) \sum_{n \geq 0}(-1)^{n}(n+1)\left(\frac{y^{2}}{r^{2}}-2 y \frac{\hat{\mathbf{x}} \cdot \hat{\mathbf{y}}}{r}\right)^{n} \\
& =\frac{L^{4}}{r^{4}} \int d^{6} y \rho(y) \sum_{n \geq 0} \sum_{m=0}^{n}(-1)^{n+m}(n+1) \frac{2^{m} n !}{m !(n-m) !} \frac{y^{2 n-m} \cos ^{m}(\gamma)}{r^{2 n-m}}
\end{aligned}
$$


where $\hat{\mathbf{x}}$ and $\hat{\mathbf{y}}$ are unit vectors and $\cos (\gamma)=\hat{\mathbf{x}} \cdot \hat{\mathbf{y}}$. Collecting together terms of the same radial power we obtain

$$
\sum_{n \geq 0} \sum_{m=0}^{n}(-1)^{n+m}(n+1) \frac{2^{m} n !}{m !(n-m) !} \frac{y^{2 n-m} \cos ^{m}(\gamma)}{r^{2 n-m}}=\sum_{k \geq 0} \frac{y^{k}}{r^{k}} C_{k}^{(2)}(\cos \gamma)
$$

where $C_{k}^{(2)}(\cos \gamma)$ is a Gegenbauer polynomial satisfying the following differential equation

$$
\left(\left(1-z^{2}\right) \partial_{z}^{2}-5 z \partial_{z}+k(k+4)\right) C_{k}^{(2)}(z)=0
$$

Properties of the Gegenbauer polynomials are given in [21]; in particular they satisfy an orthogonality relation

$$
\int_{-1}^{1} d z\left(1-z^{2}\right)^{3 / 2} C_{k}^{(2)}(z) C_{l}^{(2)}(z)=\frac{1}{8} \pi(k+1)(k+3) \delta_{k l}
$$

and are such that

$$
C_{k}^{(2)}(1)=\frac{1}{6}(k+1)(k+2)(k+3) .
$$

Now introducing the following coordinates on the $S^{5}$

$$
d s^{2}=d \theta^{2}+\sin ^{2} \theta d \Omega_{4}^{2}
$$

$S O(5)$ singlet spherical harmonics satisfy the differential equation

$$
\frac{1}{\sin ^{4} \theta} \partial_{\theta}\left(\sin ^{4} \theta \partial_{\theta}\right) Y_{k}=-k(k+4) Y_{k}
$$

which is the same equation as (A.4) with $z=\cos \theta$. The canonically normalized spherical harmonics are therefore given by

$$
Y_{k}(\theta)=\frac{\sqrt{3}}{2^{k / 2}(k+1) \sqrt{(k / 2+1)(k+3)}} C_{k}^{(2)}(\cos \theta) \equiv \lambda_{k} C_{k}^{(2)}(\cos \theta) ;
$$

with the values on the axis $\theta=0$ being

$$
Y_{k}(0)=\frac{\sqrt{(k / 2+1)(k+3)}}{\sqrt{3} 2^{k / 2}} \equiv y_{k}
$$

Note that spherical harmonics which are not $S O(5)$ singlets take the form $Y_{k}^{p}(\theta) Y_{p k}\left(\theta_{4}\right)$ with $\theta_{4}$ coordinates on $S^{4}$ and

$$
\frac{1}{\sin ^{4} \theta} \partial_{\theta}\left(\sin ^{4} \theta \partial_{\theta}\right) Y_{k}^{p}-\frac{p(p+3)}{\sin ^{2} \theta} Y_{k}^{p}=-k(k+4) Y_{k}^{p},
$$

where $p$ is the $S O(5)$ eigenvalue (on the $S^{4}$ ) with $p \leq k$. The relevant solutions are again expressed in terms of Gegenbauer polynomials as

$$
Y_{k}^{p} \propto \sin ^{p} \theta C_{k-p}^{(p+2)}(\cos \theta)
$$


Since $C_{k-p}^{(p+2)}(1)$ is non-vanishing and finite, the total harmonic is zero when $\theta=0$. Thus only the $S O(5)$ singlet spherical harmonics are non-vanishing at $\theta=0$.

Comparing (A.2) and (A.3) with (3.3) and (3.6) implies that the following identity needs to be proved

$$
C_{k}^{(2)}(\cos \gamma)=2^{k}(k+1) \sum_{I} Y_{k}^{I}\left(\theta_{5}^{y}\right) Y_{k}^{I}\left(\theta_{5}\right)
$$

where we use the identity

$$
C_{i_{1} \cdots i_{k}}^{I} y^{i_{1}} \cdots y^{i_{k}}=y^{k} Y_{k}^{I}\left(\theta_{5}^{y}\right)
$$

with $\left(\theta_{5}^{y}\right)$ the coordinates on the $y$-sphere. Now (A.13) is the exact analogue of the addition theorem for spherical harmonics of $S O(3)$ used in electromagnetism and can be proved in exactly the same way [22]. First note that in the coordinate system (A.7) on the sphere

$$
\cos \gamma=\cos \theta \cos \theta_{y}+\sin \theta \sin \theta_{y}\left(\cos \gamma_{4}\right)
$$

where $\gamma_{4}$ is the angle separating the vectors on the $S^{4}$. Thus when $\theta_{y}=0$ (it lies on the "axis") $\cos \gamma=\cos \theta$. Since the $S O(5)$ singlet harmonic is the only harmonic at level $k$ which is non-vanishing on the axis (A.13) collapses to

$$
C_{k}^{(2)}(\cos \theta)=2^{k}(k+1) Y_{k}(0) Y_{k}(\theta)
$$

an identity which is manifestly true because of (A.9) and (A.10).

Now consider rotating the axes so that $\theta_{y}$ is no longer zero. Then the function $C_{k}^{(2)}(\cos \gamma)$ still satisfies the covariant version of (A.4), namely

$$
(\square+k(k+4)) C_{k}^{(2)}(\cos \gamma)=0,
$$

where $\square$ is the Laplacian on the $S^{5}$ with coordinates $\theta_{5}$. In other words, the function can always be expanded in spherical harmonics of rank $k$ as

$$
C_{k}^{(2)}(\cos \gamma)=\sum_{I} \alpha_{k}^{I}\left(\theta_{5}^{y}\right) Y_{k}^{I}\left(\theta_{5}\right),
$$

where the coefficients are given by

$$
\alpha_{k}^{I}\left(\theta_{5}^{y}\right)=z_{k}^{-1} \int_{S^{5}} d \Omega_{5} Y_{k}^{I}\left(\theta_{5}\right) C_{k}^{(2)}(\cos \gamma)
$$

However, a generic function can be expanded in terms of spherical harmonics as

$$
f\left(\theta_{5}\right)=\sum_{k, I} \beta_{k I} Y_{k}^{I}\left(\theta_{5}\right)
$$


where

$$
\beta_{k I}=\frac{1}{z_{k}} \int_{S^{5}} d \Omega_{5} f\left(\theta_{5}\right) Y_{k}^{I}\left(\theta_{5}\right)
$$

and in particular for the $S O(5)$ singlet coefficients

$$
\beta_{k}=\frac{\lambda_{k}}{z_{k}} \int_{S^{5}} d \Omega_{5} f\left(\theta_{5}\right) C_{k}^{(2)}(\cos \theta)
$$

so that $f(\theta=0)=\sum_{k} \beta_{k} y_{k}$. Then (A.19) is the $S O(5)$ singlet coefficient in an expansion of the function $Y_{k}^{I}\left(\theta_{5}\right) / \lambda_{k}$ in a series of $Y_{k}^{I}(\gamma, \cdots)$ (i.e. with respect to the rotated axis discussed earlier). One can thus read off the coefficient (A.19) as

$$
\alpha_{k}^{I}\left(\theta_{5}^{y}\right)=\frac{1}{y_{k} \lambda_{k}} Y_{k}^{I}\left(\theta_{5}(\gamma, \cdots)\right)_{\gamma=0}=2^{k}(k+1) Y_{k}^{I}\left(\theta_{5}^{y}\right)
$$

since in the limit $\gamma \rightarrow 0$ the angles $(\theta, \cdots)$ go over into $\left(\theta_{y}, \cdots\right)$. This completes the proof of (A.13) and hence of (3.6). Note that (A.13) also implies a sum rule for harmonics of the same degree:

$$
\sum_{I}\left(Y_{k}^{I}\left(\theta_{5}\right)\right)^{2}=2^{-k}(1+k / 2)(1+k / 3)
$$

analogous to that sometimes used in electromagnetism.

\section{References}

[1] J. M. Maldacena, "The large N limit of superconformal field theories and supergravity," Adv. Theor. Math. Phys. 2, 231 (1998) [Int. J. Theor. Phys. 38, 1113 (1999)] [arXiv:hep-th/9711200].

[2] S. S. Gubser, I. R. Klebanov and A. M. Polyakov, "Gauge theory correlators from non-critical string theory," Phys. Lett. B 428, 105 (1998) [arXiv:hep-th/9802109].

[3] E. Witten, "Anti-de Sitter space and holography," Adv. Theor. Math. Phys. 2, 253 (1998) [arXiv:hep-th/9802150].

[4] O. Aharony, S. S. Gubser, J. M. Maldacena, H. Ooguri and Y. Oz, "Large N field theories, string theory and gravity," Phys. Rept. 323, 183 (2000) [arXiv:hep-th/9905111].

[5] E. D'Hoker and D. Z. Freedman, "Supersymmetric gauge theories and the AdS/CFT correspondence," [arXiv:hep-th/0201253].

[6] P. Kraus, F. Larsen and S. P. Trivedi, "The Coulomb branch of gauge theory from rotating branes," JHEP 9903, 003 (1999) [arXiv:hep-th/9811120]. 
[7] I. R. Klebanov and E. Witten, "AdS/CFT correspondence and symmetry breaking," Nucl. Phys. B 556, 89 (1999) [arXiv:hep-th/9905104].

[8] D. Z. Freedman, S. S. Gubser, K. Pilch and N. P. Warner, "Continuous distributions of D3-branes and gauged supergravity," JHEP 0007, 038 (2000) [arXiv:hep-th/9906194].

[9] S. de Haro, S. N. Solodukhin and K. Skenderis, "Holographic reconstruction of spacetime and renormalization in the AdS/CFT correspondence," Commun. Math. Phys. 217, 595 (2001) [arXiv:hep-th/0002230].

[10] M. Bianchi, D. Z. Freedman and K. Skenderis, "How to go with an RG flow," JHEP 0108, 041 (2001) [arXiv:hep-th/0105276].

[11] M. Bianchi, D. Z. Freedman and K. Skenderis, "Holographic renormalization," Nucl. Phys. B 631, 159 (2002) [arXiv:hep-th/0112119].

[12] K. Skenderis and M. Taylor, "Kaluza-Klein holography," to appear in JHEP, [arXiv:hep-th/0603016].

[13] H. J. Kim, L. J. Romans and P. van Nieuwenhuizen, "The Mass Spectrum Of Chiral $\mathrm{N}=2 \mathrm{D}=10$ Supergravity On $S^{5}$," Phys. Rev. D 32, 389 (1985).

[14] S. M. Lee, S. Minwalla, M. Rangamani and N. Seiberg, "Three-point functions of chiral operators in D =4, N =4 SYM at large N," Adv. Theor. Math. Phys. 2, 697 (1998) [arXiv:hep-th/9806074].

[15] K. Skenderis, "Lecture notes on holographic renormalization," Class. Quant. Grav. 19, 5849 (2002) [arXiv:hep-th/0209067].

[16] I. Papadimitriou and K. Skenderis, "AdS / CFT correspondence and geometry," in *Strasbourg 2003, AdS/CFT correspondence* 73-101, [arXiv:hep-th/0404176]; "Correlation functions in holographic RG flows," JHEP 0410, 075 (2004) [arXiv:hepth/0407071].

[17] H. Lin, O. Lunin and J. Maldacena, "Bubbling AdS space and 1/2 BPS geometries," JHEP 0410 (2004) 025 [arXiv:hep-th/0409174].

[18] K. A. Intriligator, "Maximally supersymmetric RG flows and AdS duality," Nucl. Phys. B 580, 99 (2000) [arXiv:hep-th/9909082]. 
[19] S. S. Gubser, A. Hashimoto, I. R. Klebanov and M. Krasnitz, "Scalar absorption and the breaking of the world volume conformal Nucl. Phys. B 526, 393 (1998) [arXiv:hepth/9803023].

[20] S. S. Gubser and A. Hashimoto, "Exact absorption probabilities for the D3-brane," Commun. Math. Phys. 203, 325 (1999) [arXiv:hep-th/9805140].

[21] M. Abramowitz and I. A. Stegun, "Handbook of mathematical functions with formulas, graphs, and mathematical tables", Dover, 1972, see chapter 22.

[22] J. D. Jackson, "Classical Electrodynamics", 2nd Edition, Wiley, see the discussion in section 3.6. 